\title{
Consecutive Ring-Expansion Reactions for the Iterative Assembly of Medium-Sized Rings and Macrocycles
}

\author{
Thomas C. Stephens \\ William P. Unsworth* (D) \\ University of York, Department of Chemistry, York, Y010 5DD, UK \\ william.unsworth@york.ac.uk \\ Published as part of the Cluster Iterative Synthesis
}

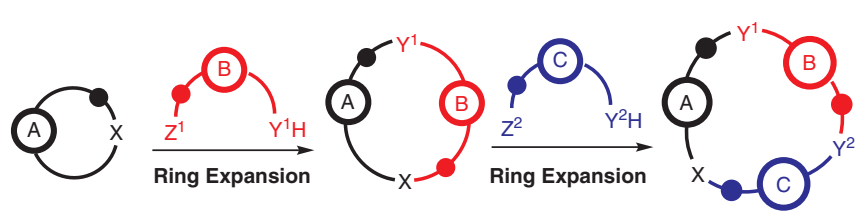

Received: 04.03.2019

Accepted after revision: 26.03.2019

Published online: 12.04 .2019

DOI: 10.1055/s-0037-1611500; Art ID: st-2019-k0130-c

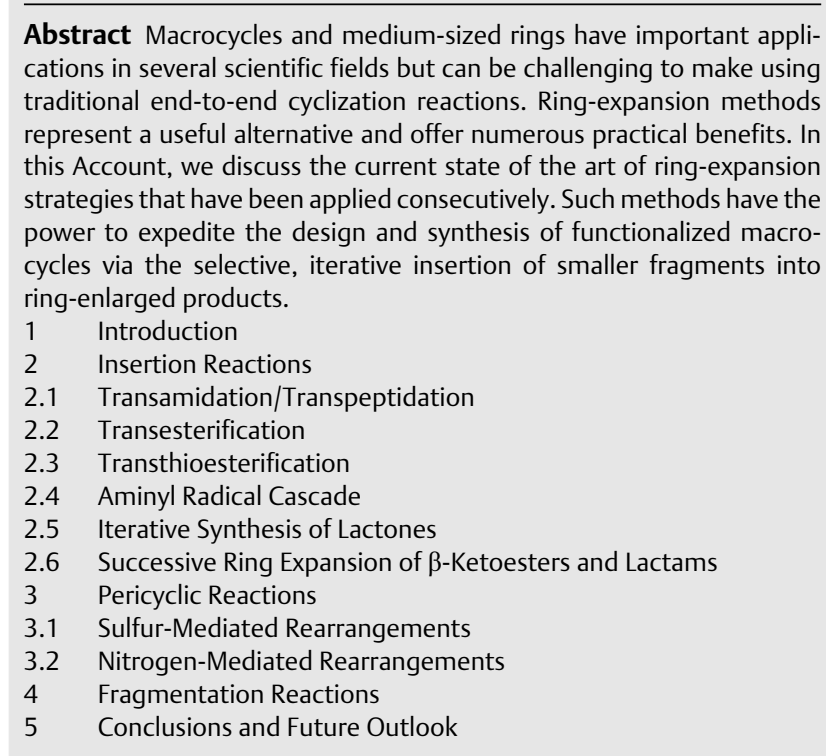

Key words ring expansion, ring enlargement, rearrangements, macrocycles, medium-sized rings, iterative synthesis

\section{Introduction}

Medium-sized rings and macrocycles are important in a wide array of scientific fields and technologies, ${ }^{1,2}$ propagating the development of several methods for their synthesis. Most of these synthetic methods are based on the end-toend cyclization of a linear precursor, which is often a challenging process; ${ }^{3}$ achieving efficient cyclization whilst avoiding unwanted intermolecular reactions such as dimerization or polymerization is usually the main problem to overcome. Various innovative strategies to help favor cyclization have emerged over the years, including the use of high-dilution conditions, ${ }^{4}$ pseudo-high-dilution conditions, ${ }^{5}$ templated systems, ${ }^{6}$ and the incorporation of internal structural features to help bias conformation towards cyclization, ${ }^{7}$ amongst others. ${ }^{8}$

All these methods are based on improving the efficiency of an often-difficult long-chain end-to-end cyclization step. Ring-expansion approaches are different as they allow this difficult step to be avoided completely. ${ }^{9,10}$ Consequently, several creative ring-expansion processes have been developed and used to facilitate the synthesis of medium-sized rings and macrocycles, with this topic reviewed by our group in $2017.9^{\mathrm{b}}$ Synthetic methods where ring-expansion reactions have been applied consecutively are far less common. Nonetheless, those that have been reported clearly demonstrate the high potential of such strategies for the iterative construction of large ring molecules.

The current state-of-the-art in the application of consecutive ring-expansion strategies is discussed in this Account. Our intention is to highlight the power of 'growing' large ring systems via the sequential or iterative insertion of smaller linear fragments. We hope that bringing together the various strategies described herein will help to propagate the use of iterative ring-expansion processes in synthetic chemistry, ${ }^{11}$ and in turn will enable functional large ring molecules to be designed with greater freedom and ambition than is possible using traditional cyclization approaches. The procedures are grouped into three main categories of ring expansion based on 'insertion', 'pericyclic', or 'fragmentation' reactions, and we have decided to predominantly focus on methods where the ring size is increased by two or more atoms in each iteration. As this Account is focused on fully controlled ring-expansion approaches, methods for the synthesis of cyclic polymers via ring-expansion polymerization have not been included. ${ }^{12}$ 


\section{Insertion Reactions}

The most common class of ring-expansion reaction used as part of a consecutive ring-enlargement sequence are side-chain insertion reactions. Within this subcategory, transamidation- and transesterification-type processes appear most frequently.

\subsection{Transamidation/Transpeptidation}

Hesse (unquestionably one of the all-time greats in the ring expansion field) and co-workers ${ }^{13}$ pioneered a series of ring expansions coined 'zip reactions' that enable polyamine-based macrocycles to be prepared via innovative cascade processes. A prototypical example of this class of reaction is illustrated in Scheme 1; thus, sodiated laurolactam 1 can be alkylated with acrylonitrile, reduced to form amine $\mathbf{2}$, and then alkylated and reduced again in the same way to form diamine 3a. The treatment of 3a under strongly basic conditions (using KAPA = potassium 3-aminopropylamide) at reflux then sets up an equilibrium in which ring expansion (3a $\rightarrow \mathbf{3 b}$ ) and ring expansion again $(\mathbf{3 b} \rightarrow \mathbf{4})$ is achieved via two sequential acyl transfer reactions. Even more impressively, there is no need to stop at just two expansions, provided the requisite starting material can be prepared; for example, 33-membered polyamine lactam $\mathbf{5}$ was synthesized in high yield using the same approach (this time via five sequential ring-expansion reactions).

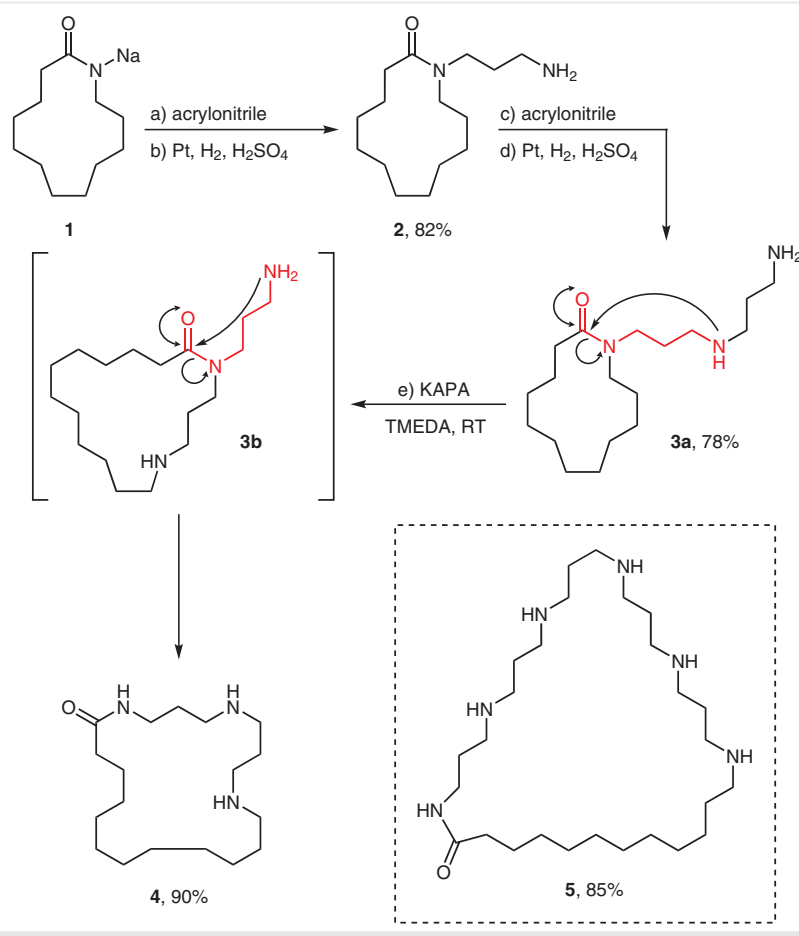

Scheme 1 'Zip' reactions developed by Hesse et al.

The Hesse group went on to establish a significant body of work in this area, ${ }^{9 a}$ with projects based on both extending the synthetic methodology and in natural product target synthesis. Arguably, the most striking example was

\section{Biographical Sketches}
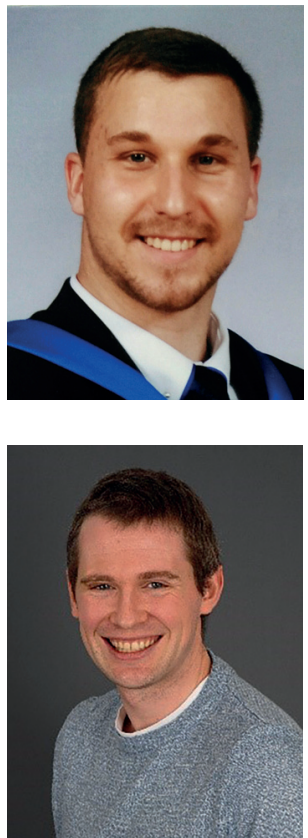

Thomas C. Stephens achieved his Master's degree at the University of Warwick in 2016. During his undergraduate studies, Tom performed a three-month placement under the supervision of Prof. Nuno Maulide at Universität Wien and completed his Masters research project with Dr. Graham Pattison. He then joined Dr. William P. Unsworth at the University of
York, where he is currently completing his Ph.D. studies. His current research interests include iterative homologation and ring expansion reactions.
Dr William P. Unsworth is an independent research fellow (Leverhulme Trust funded) at the University of York. His two main research interests are the synthesis of spirocycles using catalytic dearomative approaches and macrocycle synthesis using iterative ring expansion reactions. His work in both of these areas has been recognised by several prizes and awards, most notably the 2018 RSC Hickinbottom Award. 
their synthesis of 54-membered macrocycle 7 in 38\% yield from a 13-membered lactam polyamine derivative 6 (Scheme 2). ${ }^{14}$

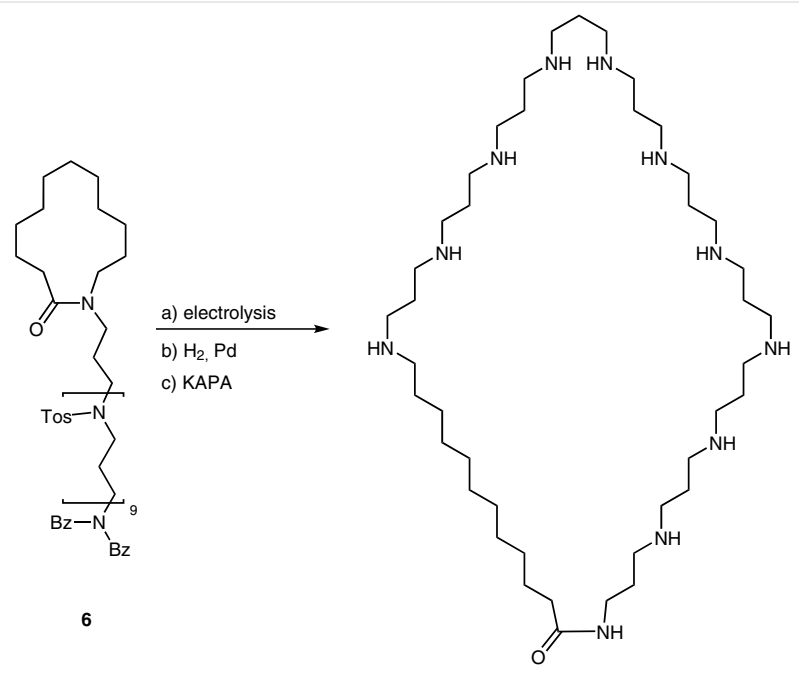

7, $38 \%$ for step c)

Scheme 2 'Zip' reaction to prepare 54-membered polyamine macrocycle 7

The 'zip reactions' described above all start from macrocyclic precursors. Ring expansion of smaller rings (e.g., 5-7membered rings) can often be more challenging in comparison, especially when the reactions are under thermodynamic control and the desired products are medium-sized rings, as these ring systems often suffer from destabilizing transannular interactions. In some cases, the problems associated with the instability of medium-sized ring products can be overcome by performing consecutive ring-expansion reactions in one-pot. An instructive example concerns the double ring expansion of 6 -membered ring barbiturate 8a into 14-membered product $\mathbf{9}$. In this case, the intermediate 10-membered ring $\mathbf{8 b}$ is unstable with respect to ring contraction back to its 6-membered precursor, however, as a second ring expansion is possible, an equilibrium is established in which 14-membered ring product $\mathbf{9}$ is formed, albeit in modest yield (Scheme 3). Consecutive ring-expansion cascade sequences like this, enable ring-enlarged products to be formed via unstable intermediates such as medium-sized ring $\mathbf{8 b}$ that would be very challenging to access and isolate directly. ${ }^{9 a}$
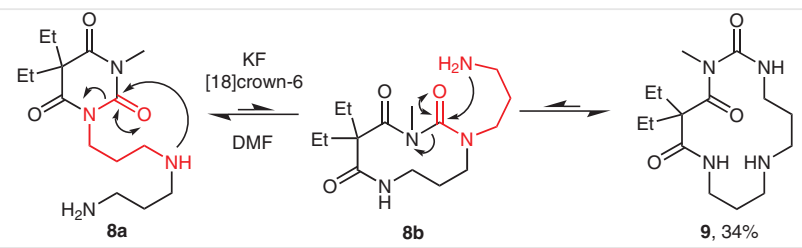

Scheme 3 Reversible consecutive 6- to 10- to 14-membered ring expansion/contraction
An early example of similar reactivity being used during a natural product synthesis can be found in the synthesis of desoxo-indandenine, ${ }^{15}$ a member of the spermidine family of natural products (Scheme 4). Starting from 13-membered cyclic ketone 10, $\alpha$-nitration was achieved via an enol acetate intermediate, and this followed by a conjugate addition reaction of $\mathbf{1 1}$ into acryladehyde. Reductive amination of the resulting aldehyde $\mathbf{1 2}$ with partially protected triamine then afforded ketone 13, which was found to exist in equilibrium with ring-expanded amide 14 under the conditions used for its formation. Switching to mildly basic, aqueous conditions was sufficient to drive the equilibrium towards the formation of the desired ring-expanded product 14, which was isolated in 55\% yield. Next, hydrolysis and a three-step reductive Nef reaction sequence removed the nitro group to form lactam 16. Finally, electrolysis was used to remove the tosyl groups, and treatment of the resulting diamine with $p$-toluenesufonic acid (PTSA) at reflux in xylene produced a 1:1 mixture of 17- and 21-membered lactams 17 and 18. This sequence demonstrates the power of transamidation reactions for the construction of complex aza-macrocyclic scaffolds. However, it also highlights that

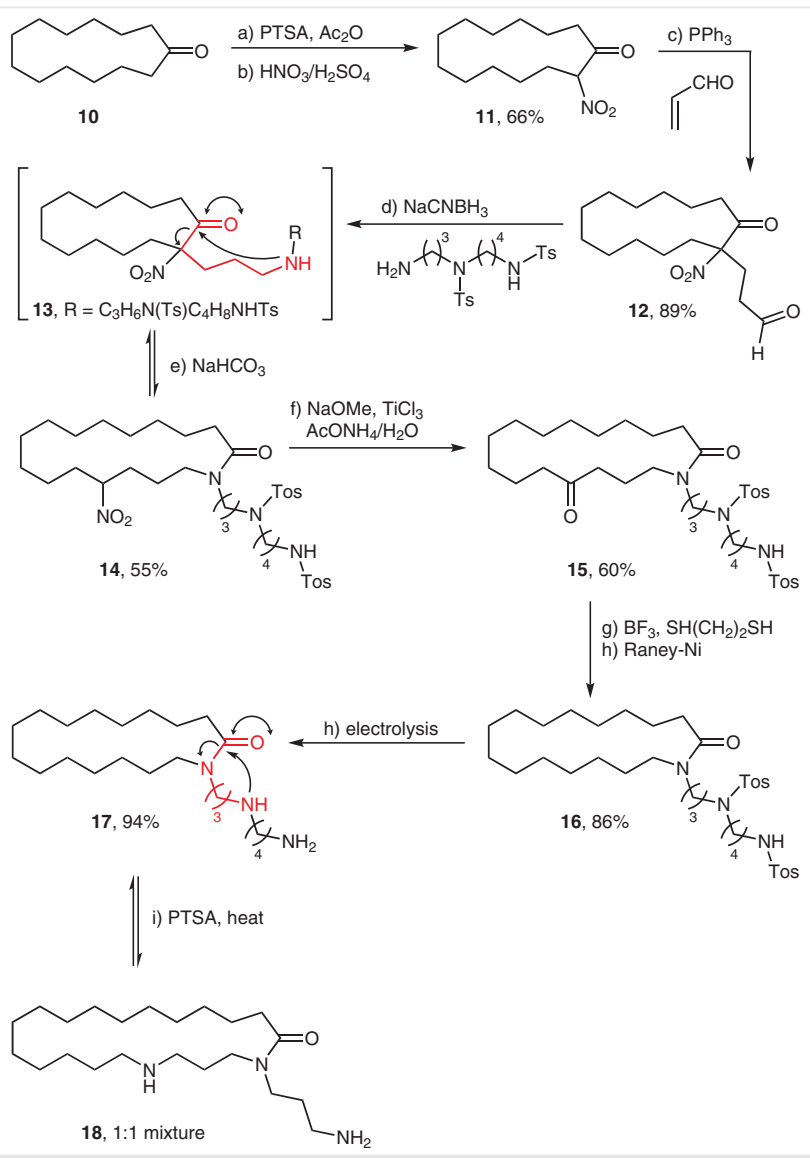

Scheme 4 Synthesis of 21-membered lactam 18 including two reversible transamidation ring-expansion reactions 
the reversibility of the transamidation rearrangement can be a problem, and this must be considered when designing reactions of this type; indeed, similar problems with the reversibility of the ring-expansion steps were also found in Hesse's work. ${ }^{9 a}$

Independently, Takahashi and co-workers ${ }^{16}$ published a similar transamidation approach during their synthesis of macrocyclic tetra-amines structurally related to some polyamine alkaloids, and also showed that these products are efficient Fe(II) binders in aqueous solutions. Thus, coumarin 19 was reacted with tetra-amine 20 in methanol at reflux for two weeks, and macrocyclic product 21d was produced in $20 \%$ yield (Scheme 5 ). The reaction is proposed to operate via an initial 1,4-conjugate addition, followed by an intramolecular amidation and one-atom ring expansion to liberate the tethered phenol $(\mathbf{1 9} \rightarrow \mathbf{2 1 a} \rightarrow \mathbf{2 1 b})$. A series of transamidation reactions then takes place (presumably under thermodynamic control) to relieve the strain associated with the medium-sized ring. Although not stated in this manuscript, it is possible that some of the smaller ring systems invoked in this presumed equilibrium were also formed in this reaction, which could account for some of the mass balance in this highly impressive, but modest yielding cascade reaction.

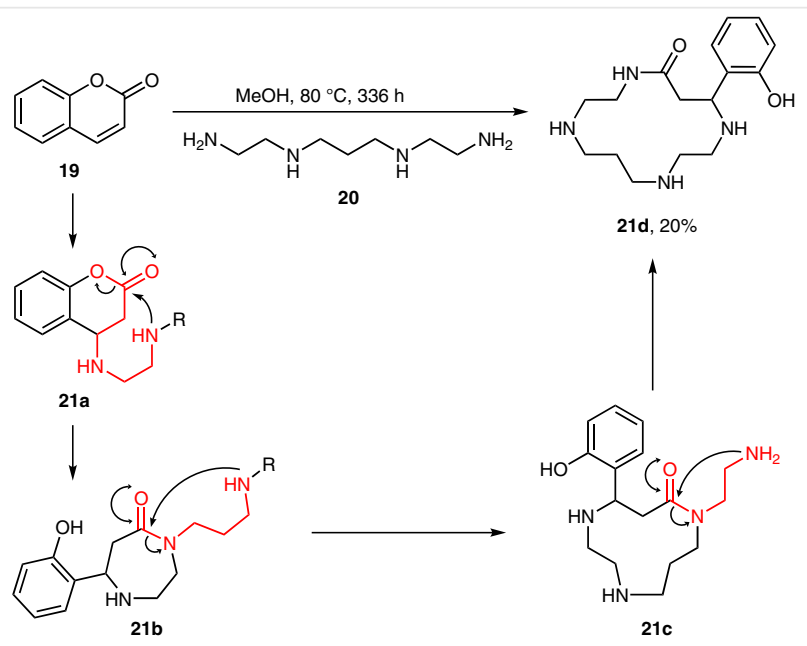

Scheme 5 Cascade ring-expansion reactions for the conversion of coumarin 19 into 14-membered lactam 21d

\subsection{Transesterification}

There is less precedent for transesterification reactions (or translactonizations) being performed consecutively in macrocycle synthesis, which perhaps reflects the relative rarity of polylactones in nature compared with polyamides (i.e., cyclic peptides are relatively common). Nonetheless, Corey and Nicolaou ${ }^{17}$ did show that reversible transesterifications processes can be performed, provided that there is a thermodynamic preference for accessing a particular ring size relative to the starting materials. Thus, 15 -membered lactone 27 was produced via two intramolecular transesterifications as shown in Scheme 6. In this work, lactonic acid 22 was converted into the corresponding pyridine thioester 23 and was reacted with Grignard reagent to produce ketolactone 24. Reduction with sodium borohydride in ethanol, followed by desilyation with TBAF in THF gave dihydroxylactone 26 a in $90 \%$ yield. Ring expansion was then promoted using catalytic PTSA to produce 15-membered lactone 27 in an impressive 90\% yield, as a 1:1 mixture of diastereoisomers (with the mixture of diastereoisomers arising during the sodium borohydride reduction step).

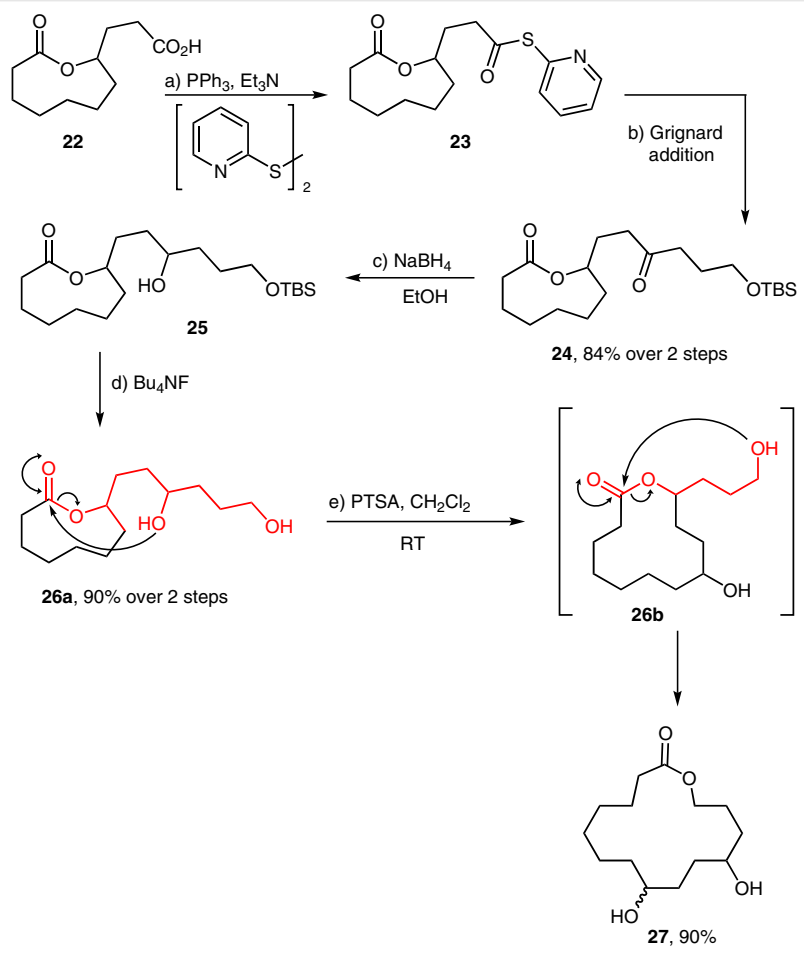

Scheme 6 A double translactonization approach to lactone $\mathbf{2 7}$

\subsection{Transthioesterification}

Inteins are sections of proteins which have the ability to excise themselves from the peptide sequence in a process called splicing. Intein splicing operates through a series of acyl shifts which is commonly facilitated by a cysteine residue which can induce an $N, S$-acyl shift to break the peptide bond. Tam and co-workers ${ }^{18}$ have developed a clever 'thiazip reaction' to produce macrocyclic peptides via a ring-expansion cascade that bears striking similarity to the biological approach used by inteins. Thus, starting from a cysteine-rich linear peptide, an initial activation step using an external thiol produces thiolactone (represented as $\mathbf{2 8}$, Scheme 7). The cysteine thiol residues then promote a series of reversible thiolactone exchange reactions as drawn, which operate at physiological $\mathrm{pH}$. The equilibrium is driven towards the ring-expanded product 29d via a final irre- 
versible $S$ - to $N$-acyl migration $(\mathbf{2 9 d} \rightarrow \mathbf{3 0}$ ) of an $N$-terminal cysteine residue, thus completing this ingenious multiple ring-expansion cascade sequence.

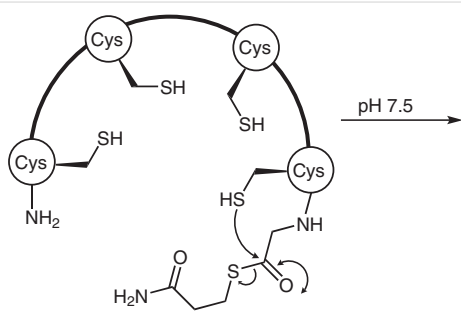

28
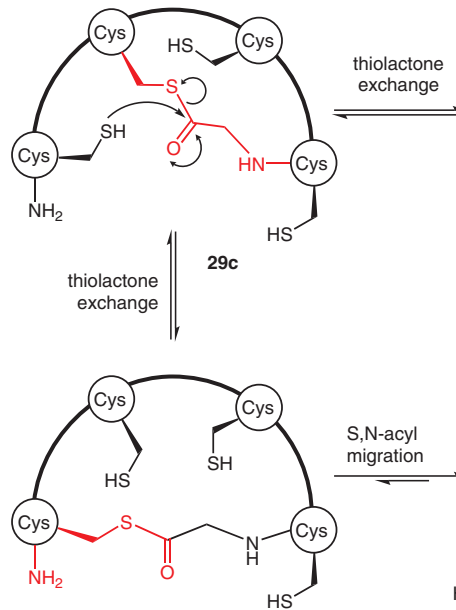

29d

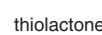
exchange
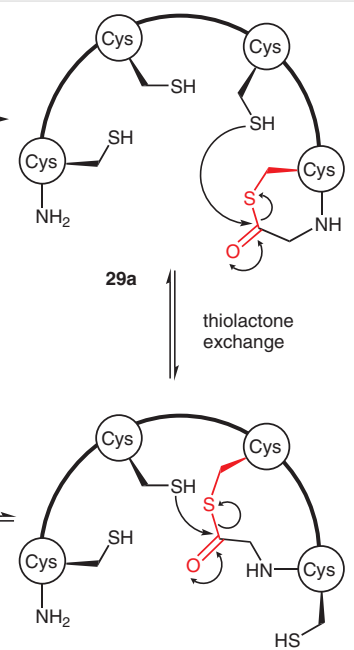

$29 b$

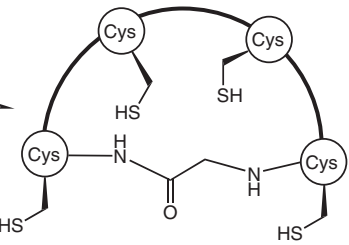

30

typical yields of $30-80 \%$

Scheme 7 A 'thia-zip' transthioesterification cascade proces

\subsection{Aminyl Radical Cascade}

Pattenden and Schulz $\mathrm{z}^{19}$ serendipitously discovered a double radical ring-expansion cascade (Scheme 8) starting from acetylene oxime 31 using (TMS) ${ }_{3} \mathrm{SiH}$ and radical initiator AIBN. It was proposed that this process is initiated by radical silylation at the terminal alkyne and that the resulting vinyl radical 32a cyclizes to form aminyl radical 32b. Fragmentation of the fused bicycle to form carbon-centered radical 32c is then followed by transannulation to the more stabilized $\alpha$-silyl radical. Cyclopropanation via a 3-exo-trig cyclization then gave 32d, which quickly ring-expanded with elimination of its silyl group, furnishing bicyclic oxime product 33. Hydrolysis of the oxime was then shown to deliver the enone in good yield.

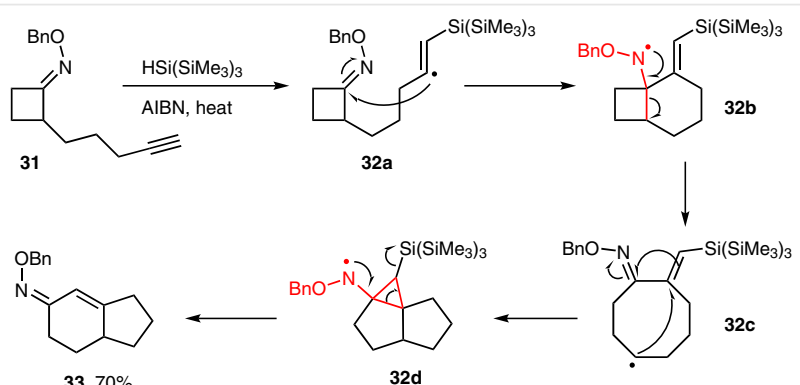

Scheme 8 Radical cascade conversion of 4-membered cyclic oxime 31 into bicyclic cyclic oxime 33

\subsection{Iterative Synthesis of Lactones}

Seyden-Penne, Rousseau, and Fouque ${ }^{20}$ developed an iterative procedure where lactones can be ring-enlarged via iterative one-carbon ring-expansion reactions through the clever use of carbenes. This is exemplified by the iterative expansion of caprolactone $\mathbf{3 4}$ (Scheme 9). First, the lactone is treated with LDA and then TMSCl to form silyl enol ether 35. Treating 35 with pre-formed ethyl carbenoid (itself made with dichloroethane and butyllithium) then yields cyclopropane 36, which upon heating at reflux in toluene undergoes desilylation and ring expansion $(\mathbf{3 6} \rightarrow \mathbf{3 7})$. Olefin hydrogenolysis is then required before another ring-enlargement iteration can be performed $(\mathbf{3 7} \rightarrow \mathbf{3 8})$. This then gives an 8-membered lactone that itself can be converted into a silyl enol ether and re-enter this ring-expansion sequence, allowing subsequent iterations of the same process to be performed (either with the same or different chlorocarbene reagents), thus enabling 9- and 10-membered lactones $\mathbf{3 9}$ and $\mathbf{4 0}$ to be synthesized in the same way. Note that while we set out to only include ring-expansion processes where the ring is enlarged by two or more atoms, we feel that the elegance and simplicity of this iterative process justified its inclusion in this Account as a notable exception.

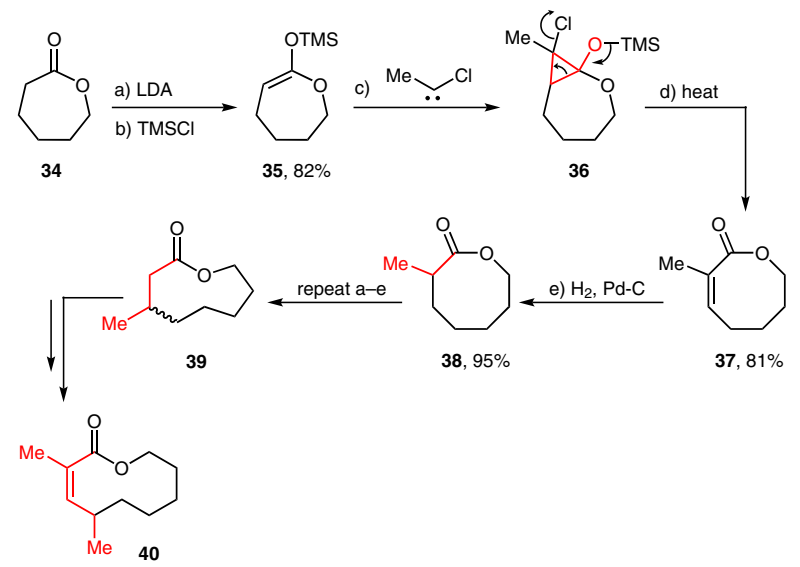

Scheme 9 Iterative one-carbon ring expansion of lactones using carbenes 


\subsection{Successive Ring Expansion of $\beta$-Ketoesters and Lactams}

In our laboratory, we have developed a system based on the successive ring expansion of cyclic $\beta$-ketoesters that allows 3- or 4-atom amino acid or hydroxy acid fragments to be inserted into ring-enlarged products iteratively. ${ }^{21}$ This process is exemplified by an iterative triple ring-expansion reaction sequence, starting from 12 -membered $\beta$-ketoester 41 (Scheme 10, a). Thus, the starting $\beta$-ketoester 41 was treated with amino acid chloride 42 in the presence of magnesium(II)chloride and pyridine to promote $C$-acylation and form tri-carbonyl species 43. Cleavage of the Fmoc protecting group ( $\mathbf{4 3} \rightarrow \mathbf{4 4 a}$ ) then initiates spontaneous cyclization $(44 a \rightarrow 44 b)$ and ring expansion $(44 b \rightarrow 45)$ in high yield over the acylation/deprotection/rearrangement sequence. As the 16-membered product $\mathbf{4 5}$ is another cyclic $\beta$-ketoester, it is itself a suitable substrate for further ring expansion and can be subjected to the same sequence to produce 20-membered and 24-membered macrocyclic products 46 and 47 via two further iterations. There is considerable freedom to vary the ring size of the starting material and use both $\alpha$ - and $\beta$-amino acids. Indeed, by varying the linear fragment, it is possible to prepare ringexpanded $\beta$-ketoester by the insertion of different functional groups in sequence (e.g., macrocycles $\mathbf{4 8}$ and $\mathbf{4 9}$, Scheme $10, \mathrm{~b})$. In a later study, the same chemistry was used to create a library of leadlike medium-sized ring scaffolds for inclusion in a high-throughput-screening compound collection. $^{22}$

The same idea can also be applied to hydroxy acid derived linear fragments, allowing ring-expanded lactones to be prepared in a similar way; in these reactions, the protected amine is replaced by a benzyl-protected alcohol that is revealed by hydrogenolysis to initiate ring expansion. Indeed, both methods can be readily combined to allow mixed lactam/lactone products (e.g., 53-55, Scheme 11) to be prepared.

We more recently extended this methodology to change the $\beta$-ketoester moiety to a simple lactam functionality. ${ }^{23}$ Using 13-membered amide 56, $\mathrm{N}$-acylation was achieved using modified thermodynamic conditions with amino acid chloride 57. The amine moiety of imide $\mathbf{5 8}$ was then revealed using $\mathrm{DBU}$, and under these conditions the amine that was formed spontaneously rearranged $(\mathbf{5 9 a} \rightarrow \mathbf{5 9 b} \rightarrow$ $\mathbf{6 0}$ ) to furnish 17-membered ring-expanded product $\mathbf{6 0}$ in excellent yield. This product can then undergo another two iterations using the same chemistry to afford both 21 - and 25-membered macrocycles (61 and $\mathbf{6 2}$, respectively, Scheme 12). A broad array of amino acids and peptoid monomers are compatible with this method, which also works well successively, enabling the synthesis of a range of tri-peptide mimetics. Note that a conceptually related ring-

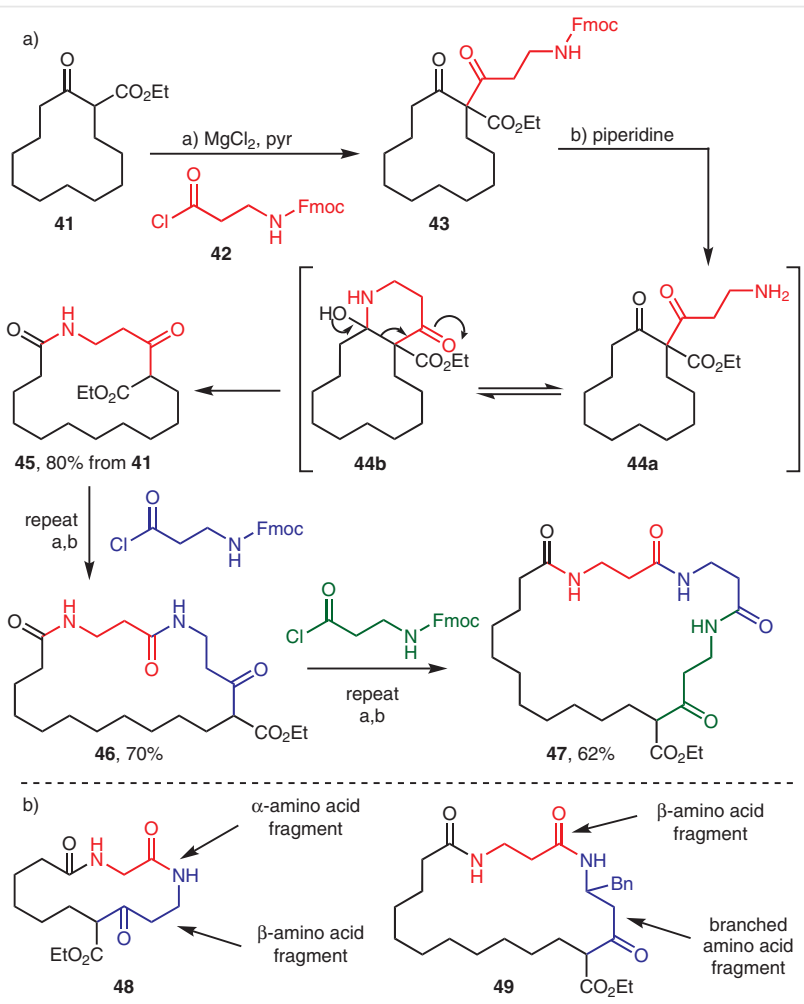

Scheme 10 Successive ring-expansion (SURE) reactions of cyclic $\beta$-ketoesters with amino acid derivatives

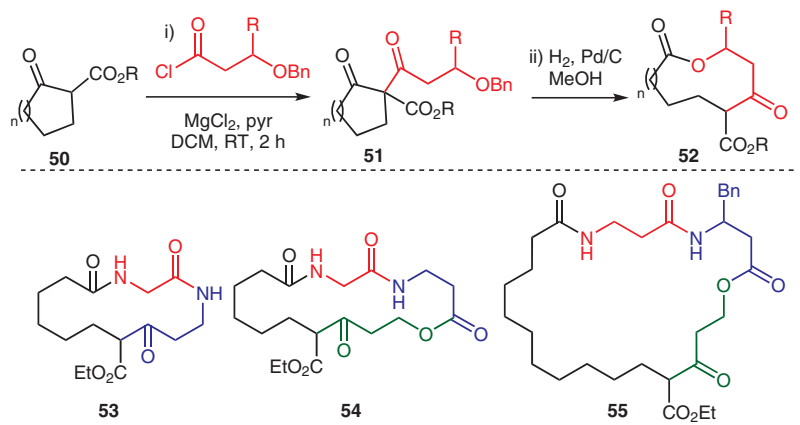

Scheme 11 Successive ring-expansion (SURE) reactions of cyclic $\beta$-ketoesters with hydroxy acid derivatives

expansion method to this one was independently developed and published around the same time by Yudin and coworkers. ${ }^{10 \mathrm{c}}$

The most recent extension to this chemistry involves the incorporation of hydroxy acids into lactam starting materials, allowing ring-expanded lactones to be made via a similar strategy. ${ }^{24}$ For example, $N$-acylation of 8 -membered lactam 63 with hydroxy acid chloride 64 can be achieved using the same acylation conditions as before, and hydrogenolysis can then be used to cleave the benzyl protecting group. Unlike the analogous amino acid chemistry, spontaneous ring expansion did not ensue in this system, instead an equilibrium was established between imide $\mathbf{6 6} \mathbf{a}$, fused 


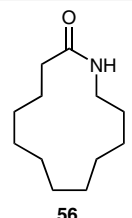

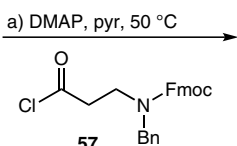

$57 \quad \mathrm{Bn}$

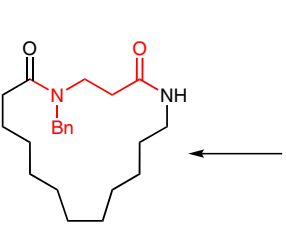

60, $91 \%$ over 2 steps<smiles>CCCCCCCCCCCC</smiles><smiles>O=C1CCN(Cc2ccccc2)C(=O)CCN(Cc2ccccc2)CCCCCCCCCCCCCCCCCCCCCN1</smiles>
$61,81 \%$

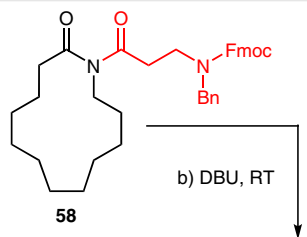

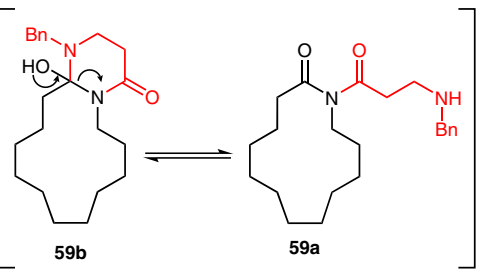
repeat
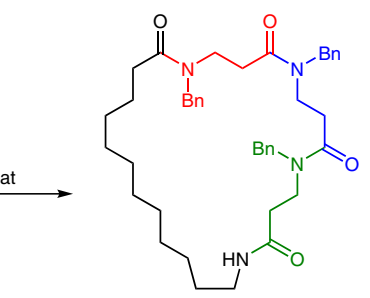

$62,77 \%$
Scheme 12 Successive ring-expansion (SuRE) reactions of lactams with amino acid derivatives

bicyclic intermediate $\mathbf{6 6 b}$, and ring-expanded product $\mathbf{6 7}$, but pleasingly, a solvent switch to chloroform and the addition of triethylamine was successful in driving the equilibrium towards the desired ring-expanded lactone 67, which was isolated in high yield (Scheme 13, a). A range of both $\alpha-$ and $\beta$-hydroxy acids are well tolerated and crucially, this method and our earlier amino acid ring-expansion reactions are fully compatible, meaning that mixed lactam/lactone macrocycles can be prepared via the iterative insertion of either functionality; selected examples of scaffolds prepared in this way are highlighted in Scheme 13 (b). The iterations can also be telescoped into an 'assembly line' type process, in which chromatography is not performed until after the final iteration; for example, 8-membered lactam 63 was taken through three iterations of the typical hydroxy acid ring-expansion procedure to form macrocycle $\mathbf{7 3}$ in $48 \%$ yield over the entire sequence (Scheme 13, c).

The SuRE methods described above are believed to be under thermodynamic control, and ring size has been shown to play a key role in their outcomes. For example, for the hydroxy acid chemistry described in Scheme 13, only lactams of 8-members or above undergo ring expansion using $\alpha$-hydroxy acids, whereas for $\beta$-hydroxy acids, lactams of 6-members or above work well. A relatively simple density functional theory (DFT) study has been performed on these reactions in which it was found that when considering the three isomeric species (e.g., 66a, 66b, and 67) presumed to be in equilibrium in these reactions, there is strong correlation between the reaction outcome and their<smiles>O=C1CCCCCCC1</smiles><smiles>CCCCC(C)C1CCCCC(=O)N(C(=O)CCOCc2ccccc2)C1</smiles>

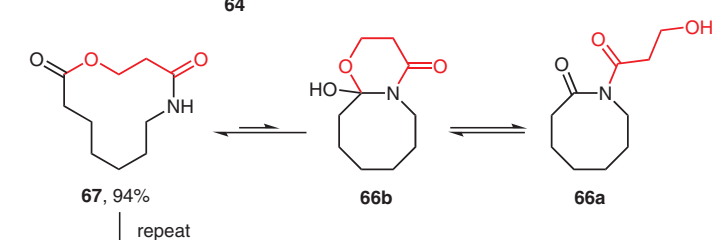<smiles>COC(=O)CCOC(=O)CCCCCCCNC(=O)CO</smiles><smiles>CCCCCCCCCC(=O)OCCC(=O)OCCC(=O)OCC(=O)NCC</smiles>

$68,84 \%$ over 2 steps 69, $84 \%$ over 2 steps

b)

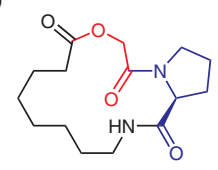

70

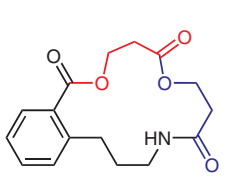

71

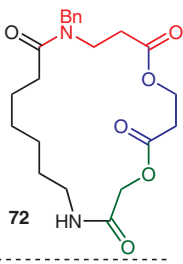

c)
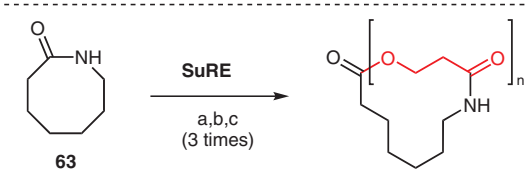

$67, \mathrm{n}=1$

$68, \mathrm{n}=2$

73, $48 \%$ over 3 iterations

Scheme 13 Successive ring-expansion (SuRE) reactions of lactams with hydroxy acid derivatives

DFT-calculated ground-state energies. For example, for the 5-membered system (which did not ring-expand) imide 74a was calculated to be significantly lower in energy than either isomer $\mathbf{7 4 b}$ or $\mathbf{7 4 c}$, whereas for the successful 8membered example, the ring-expanded product $\mathbf{7 5 c}$ was calculated to be the lowest in energy and was indeed isolated in good yield in the synthetic reaction (Scheme 14). This method used a B3LYP/6-31G* DFT method; a more rigorous and detailed DFT study on these and related reactions is currently ongoing and will be reported in due course.

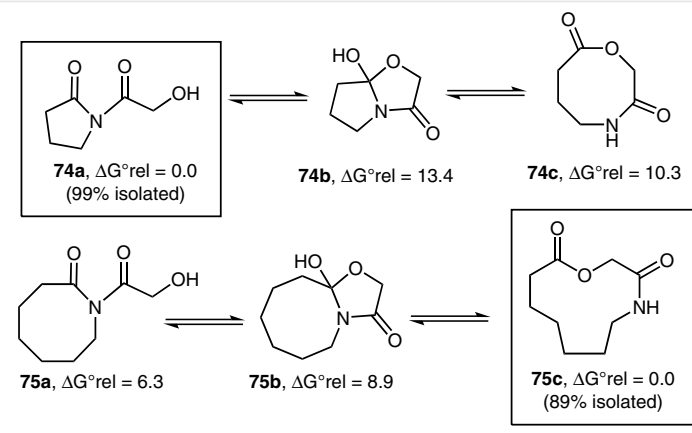

Scheme 14 DFT study of SuRE reactions 


\section{Pericyclic Reactions}

There has been a significant amount of work done on sequential sigmatropic rearrangements. These rearrangements typically require a strong thermodynamic driving force to avoid the formation of multiple products in equilibrium and have often been used in natural product synthesis.

\subsection{Sulfur-Mediated Rearrangements}

Sulfur-mediated ring-expansion reactions are often based on ylide reactivity, which can be advantageous as charged pericyclic rearrangements often proceed faster than their neutral counterparts. This is exemplified by some impressive work from Vedejs and Reid, ${ }^{25}$ who used a 2,3-sigmatropic rearrangement as part of their synthesis of the carbocyclic cytochalasin natural products, to construct a key 11-membered ring (Scheme 15). Thus, iodide 76 was heated in acetonitrile $/ \mathrm{K}_{2} \mathrm{CO}_{3}$ to form sulfonium ylide 77 , which spontaneously underwent a 2,3-sigmatropic rearrangement to form $\mathbf{7 8}$. Then, a second ring-expansion reaction was performed via methylation of sulfide 78 using Meerwein salt followed by treatment with $\mathrm{Zn} /$ acetic acid to cleave one of the $\mathrm{C}-\mathrm{S}$ bonds and furnish ring-expanded product 80, which was converted into the final target molecule $\mathbf{8 1}$ via three additional steps.
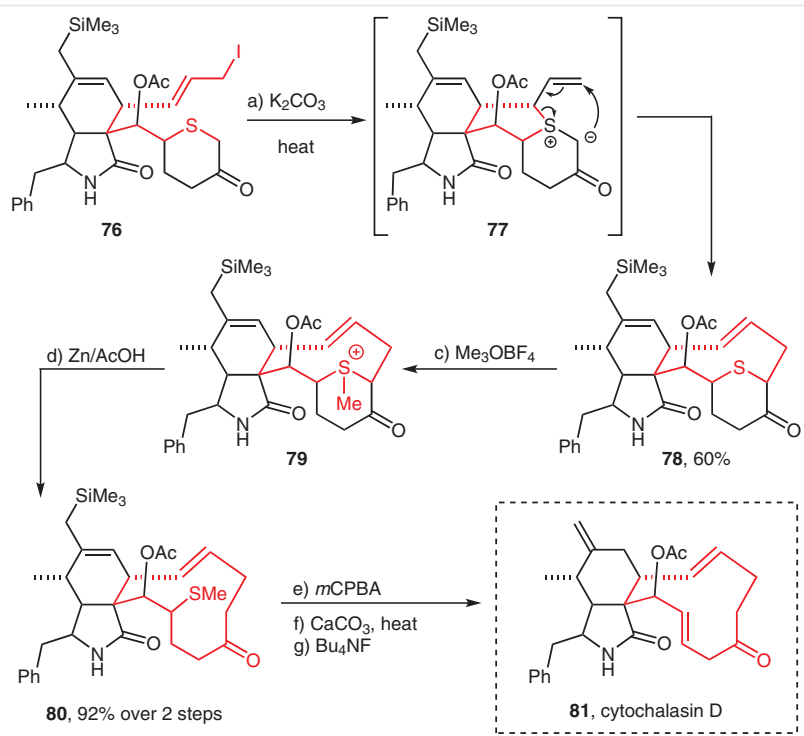

Scheme 15 Consecutive sulfur-mediated ring-expansion reactions in the total synthesis of carbocyclic cytochalasin natural product $\mathbf{8 1}$

Vedejs and co-workers were the first group to develop the idea of using consecutive 2,3-sigmatropic rearrangements (Scheme 16) in an iterative sense, ${ }^{26}$ exemplified by a sequence starting from $\alpha$-vinyl cyclic sulfide 82 . This simple 5-membered ring starting material was first converted into sulfide salt 83 upon treatment with a diazocarbonyl reagent. Then, upon addition of base, a stabilized ylide $\mathbf{8 4}$ was formed, which was found to undergo spontaneous 2,3-sigmatropic rearrangement in situ to afford ring-expanded cyclic olefin 85. A Wittig reaction was then performed to convert the ketone side chain of $\mathbf{8 5}$ into an olefin, thus furnishing new $\alpha$-vinyl cyclic sulfide $\mathbf{8 6}$ primed to enter the same ring-expansion sequence. A different diazo carbonyl species and reaction conditions were used for the second iteration, but the chemistry proceeds in broadly the same way; thus, $\alpha$-vinyl cyclic sulfide $\mathbf{8 6}$ was shown to react with diazomalonate in the presence of copper at $100{ }^{\circ} \mathrm{C}$, to form 11 membered ring product $\mathbf{8 8}$ via the same sequence of $S$-alkylation, ylide formation, and 2,3-sigmatropic rearrangement.

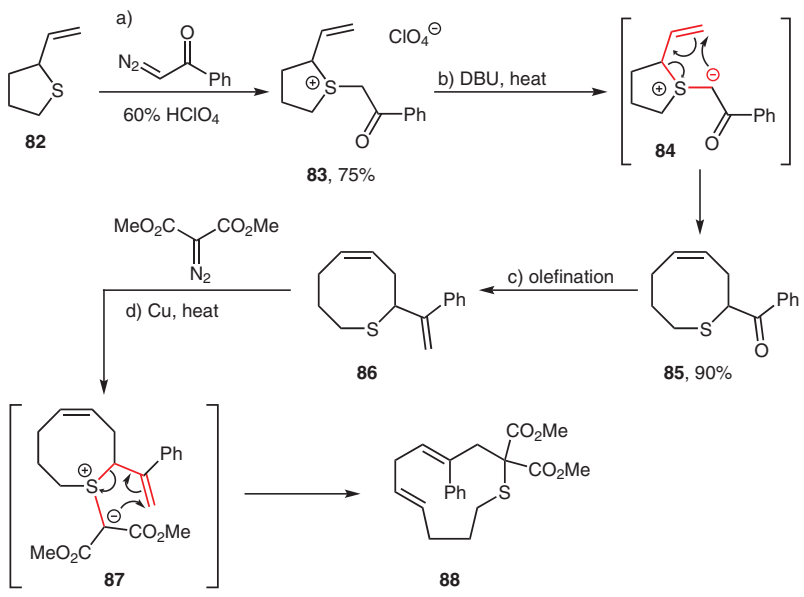

Scheme 16 Iterative 2,3-sigmatropic rearrangements of cyclic vinyl sulfides with diazocarbonyl reagents

Using a similar strategy, Schmid and Schmid ${ }^{27}$ developed an allylation-based iterative cyclic sulfide expansion (Scheme 17). Starting from the same $\alpha$-vinyl cyclic sulfide $\mathbf{8 2}$ used in the Vedejs study, it was shown that $S$-allylation can be performed by reacting with allyl bromide under acidic conditions and that subsequent treatment with aqueous potassium hydroxide promoted ylide formation $(\mathbf{8 9} \rightarrow \mathbf{9 0})$ and 2,3-sigmatropic rearrangement $(\mathbf{9 0} \rightarrow \mathbf{9 1})$ to deliver the ring-expanded product 91. An advantage of this method compared with the previous Vedejs work is that no further manipulation of the product is required to complete additional iterations, and thus it was shown that 8-membered cyclic sulfide $\mathbf{9 1}$ could be further expanded into 11and 14-membered derivatives 94 and 95 using the same method. 


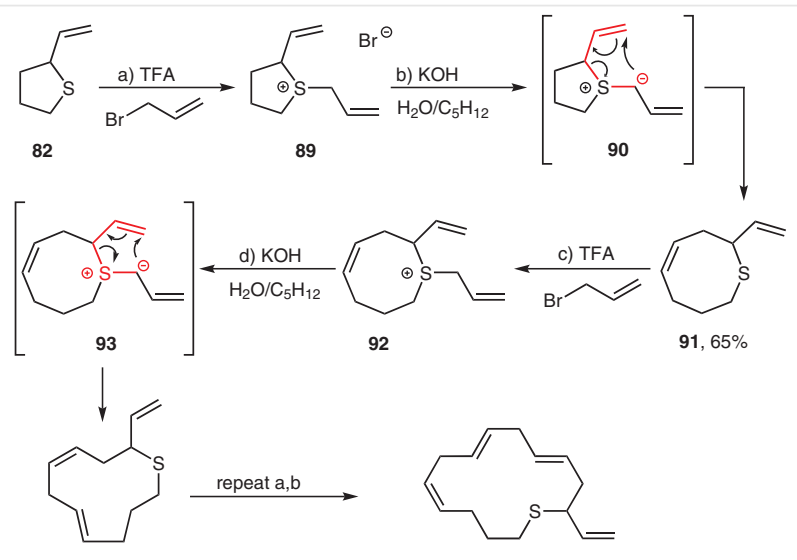

94, $76 \%$ over 2 steps

Scheme 17 Iterative 2,3-sigmatropic rearrangements of cyclic vinyl sulfides via $S$-allylation

Vedejs and co-workers then went on to extend their previous work, ${ }^{28}$ and during these studies an unwanted competing 2,3-sigmatropic rearrangement was observed (shown in Scheme 18 to form 98) originating from the formation of endocyclic ylide $\mathbf{9 7} .^{28 a}$ It is stated that the undesired endocyclic ylide forms only when making mediumsized rings, which is likely to be a consequence of the additional thermodynamic difficulties associated with making medium-sized rings (e.g., transannular strain in the products and reaction transition states). This highlights the importance of achieving the correct chemo- and regioselectivity when planning iterative ring-expansion processes and also the major impact that the size of the rings involved can have on the outcomes of ring-expansion reactions.

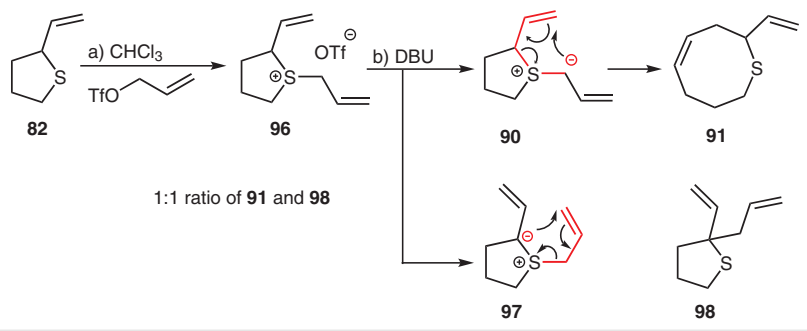

Scheme 18 Desired ring expansion and unwanted allyl transfer pathways

\subsection{Nitrogen-Mediated Rearrangements}

An interesting double 3,3-sigmatropic rearrangement approach was reported by Back and co-workers ${ }^{29}$ as part of a natural product synthesis (Scheme 19). The sequence started from 2-vinylpyrrolidine $\mathbf{9 9}$, which was reacted with acetylenic sulfone to form a zwitterionic species 100, which then underwent 3,3-aza-cope rearrangement in situ to produce ring-expanded enamine product 101. The authors then realized that this aza-Cope rearrangement could be performed for a second time if a similarly placed vinyl

group could be introduced into this product. To do this, olefin hydrogenation was followed by treatment with triflic acid and the addition of a vinyl Grignard reagent to afford 103. Removal of the tosyl group was not required to perform the next ring-expansion iteration, but it was needed for their motuporamine natural product target and was performed using a sodium-mercury amalgam to form $\mathbf{1 0 4}$. This system was then set up to undergo the same conjugate addition/aza-Cope rearrangement sequence that had previously been performed on pyrrolidine $\mathbf{9 9}$, and it was successful in enlarging the ring further to furnish 13-membered amine 106. An additional five steps were then needed to complete the total synthesis of motuporamine A.

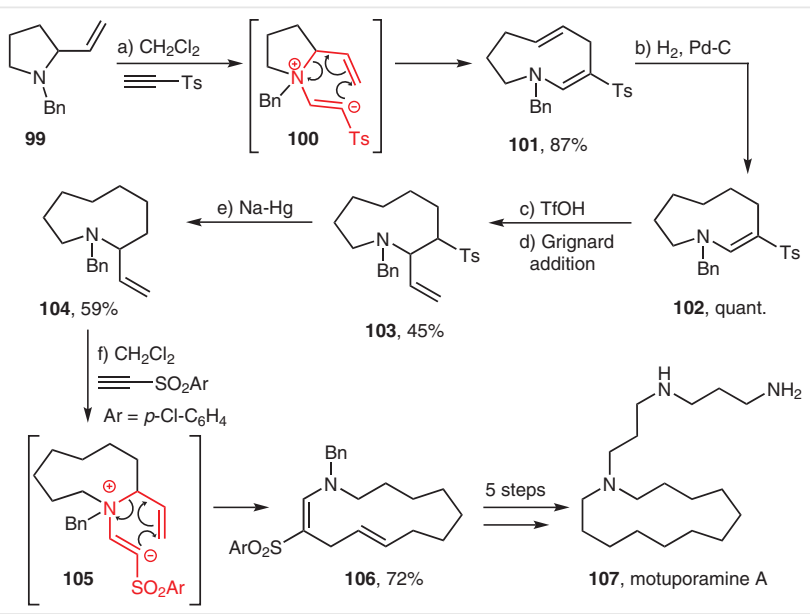

Scheme 19 Consecutive 3,3-aza-Cope rearrangement ring-expansion reactions

Suh and co-workers utilized an intriguing aza-Claisen rearrangement $(A C R)$ during their total syntheses of fluvirucinines $A_{1}$ and $A_{2}, 30,31$ in which two ring-expansion rearrangements were performed with excellent control of the stereogenic centers formed in construction of the 14-membered skeleton (Scheme 20). The sequence started from piperidinone 108, which was synthesized by an asymmetric Evans alkylation and stereoselective vinylation as described previously by the same group. Then, treatment with LiHMDS formed enolate $\mathbf{1 0 9}$ which rearranged into lactam 110 as a single stereoisomer, via a 3,3-sigmotropic rearrangement. The fact that a single product was formed suggests that the reaction proceeds via $Z$-enolate $\mathbf{1 0 9}$ as shown. Olefin hydrogenation, amine protection, and partial lactam reduction/silylation then yielded $\mathrm{N}, \mathrm{O}$-acetal $\mathbf{1 1 3}$. Then, treatment with Lewis acid and an allyl tin reagent promoted a highly stereoselective amidoalkylation and concomitant deprotection to furnish 114. Re-protection of the amine was followed by oxidative cleavage of the olefin with osmium tetraoxide and sodium periodate, and then silylation of the resulting aldehyde produced the required $(E)$ enol ether $\mathbf{1 1 6}$ for the second ACR. Finally, $N$-acylation with activated trans-pentenoic acid delivered compound $\mathbf{1 1 7}$ in 
which all the functionality needed to perform the second ACR was in place. LiHMDS was again used to form amideenolate and initiate the second iterative ACR, which was successful in forming 14-membered ring 119 with >10:1 diastereoselectivity. Further manipulation of $\mathbf{1 1 9}$ was performed to produce the final target molecules fluvirucinine $\mathrm{A}_{1}$ and $\mathrm{A}_{2}$ (not shown). ${ }^{30,31}$

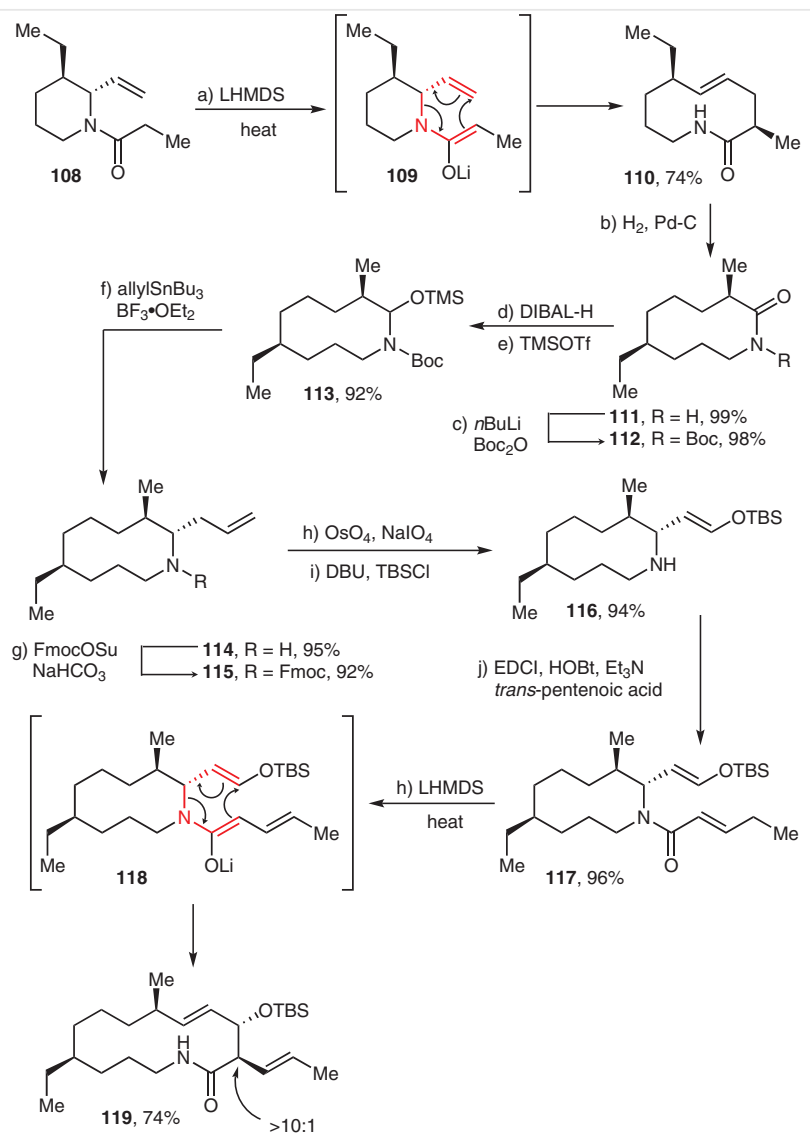

Scheme 20 Consecutive ACR reactions used in the total synthesis of fluvirucinine natural products

Later on, Suh and co-workers streamlined their synthesis of fluvirucinine $\mathrm{A}_{2}{ }^{31}$ These second-generation ACR exhibited significant kinetic improvements, and they also demonstrated the importance of the geometry of the enol ether in the ACR precursor (Scheme 21). A chair transition state is postulated to account for the large degree of stereocontrol. Further study of these enol ether precursors was completed and was well described with more details of such aza-rearrangements in a focus review. ${ }^{32}$

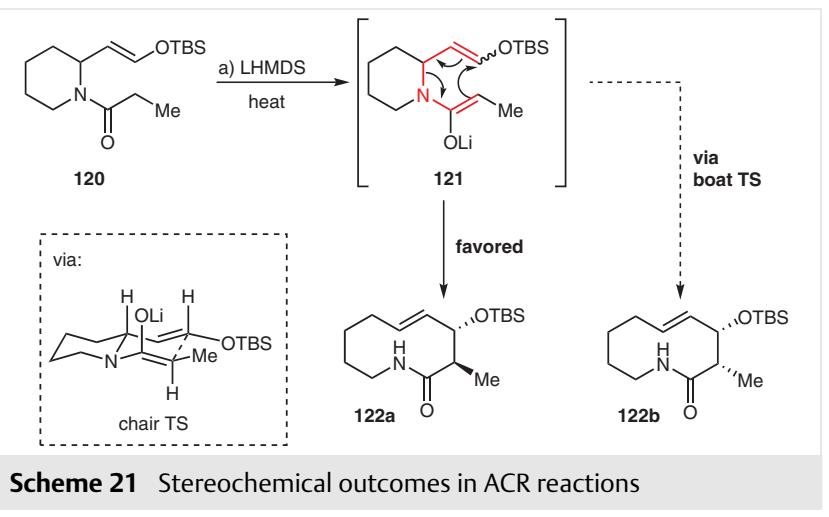

\section{Fragmentation Reactions}

Dowd and Zhang ${ }^{33}$ reported a double ring-expansion process which involves both side-chain insertion and fragmentation (Scheme 22). In this study, cyclic silyl enol ether 123 and ketene 124 were reacted via a [2+2] cycloaddition to generate cyclobutanone $\mathbf{1 2 5}$, with this reaction proceeding with excellent endo/exo selectivity when cooled to $-20{ }^{\circ} \mathrm{C}$. Classical AIBN and $\mathrm{Bu}_{3} \mathrm{SnH}$ conditions were then used to form the tributyltin radical which abstracted the pendant bromide group to generate primary radical 126a, which cyclized and fragmented $(\mathbf{1 2 6} \mathbf{a} \rightarrow \mathbf{1 2 6 b} \rightarrow \mathbf{1 2 7})$ to complete the first ring-expansion reaction. Release of ring strain in the cyclobutyl ring is presumably an important driving force in this reaction. In the same pot, radical de-chlorination (promoted by the tributyltin radical) also took place to furnish reduced compound 129. Next, lithium aluminum hydride reduction of the ketone, followed by mesylation and desilylation afforded compound 131 and set up the second ring-expansion reaction, through a base-mediated Grob fragmentation to form 11-membered ketone 132 with cis-olefin geometry in excellent yield. A similar strategy was also used to generate homologous 12-membered ketone 134 as a mixture of geometrical isomers, with a base-mediated Grob fragmentation again being a key step (see box in Scheme 22). 


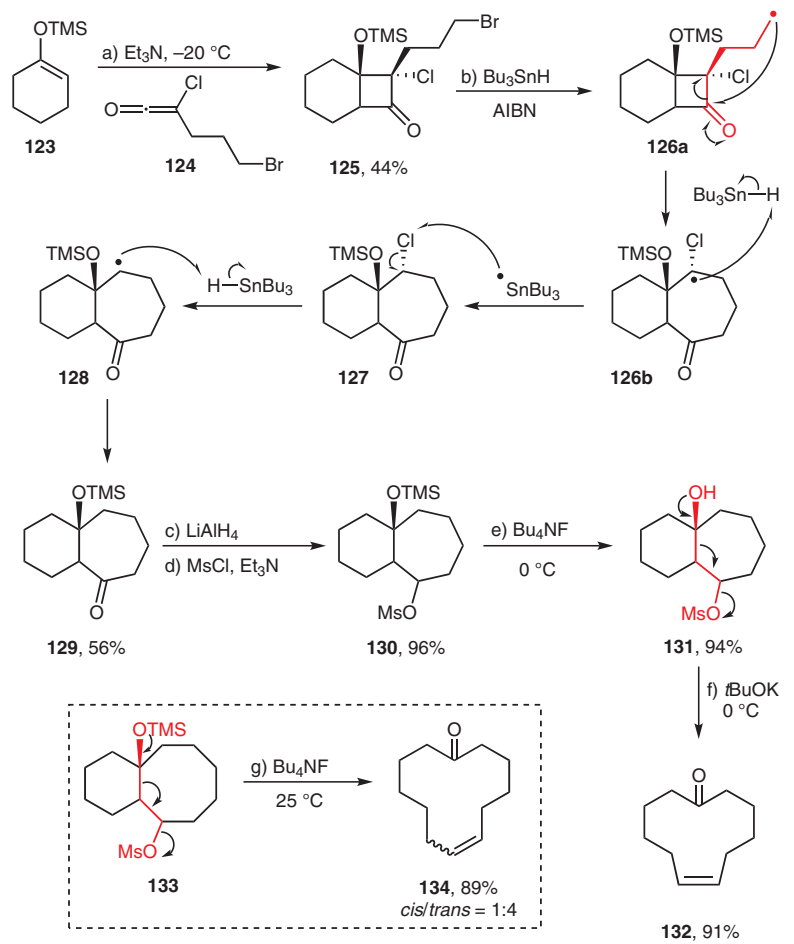

Scheme 22 A consecutive radical and Grob ring-expansion sequence

Thommen and co-workers ${ }^{34}$ accessed 15 -membered ketones via consecutive Grob fragmentations (Scheme 23), starting from tricyclic diol 135, which was itself prepared via a trimolecular aldol-type process. First, diastereoselective reduction of the ketone group of $\mathbf{1 3 5}$ to cis-triol $\mathbf{1 3 6}$ was achieved selectively using Red-Al ${ }^{\circledR}$. This was followed by tosylation using $n$-butyllithium, and the first Grob fragmentation was performed upon treatment with potassium tert-butoxide to furnish $\mathbf{1 3 8}$ as the major product. Lithium aluminum hydride reduction of $\mathbf{1 3 8}$ followed by tosylation of the secondary alcohol to form $\mathbf{1 4 0}$ then set up a second Grob fragmentation, which again was promoted by potassium tert-butoxide, to afforded 15-membered ring-expanded dienone 141.

Another impressive example of the use of sequential ring-expansion reactions involving Grob-type fragmentation was reported by Ikeda and co-workers. ${ }^{35}$ In this work, a traditional Grob-type fragmentation $(\mathbf{1 4 4} \rightarrow \mathbf{1 4 5})$ was followed by an oxidative fragmentation $(\mathbf{1 4 5} \rightarrow \mathbf{1 4 6})$, allowing an advanced precursor to the natural product 147, ( \pm )-phoracantholide $\mathrm{M}$ to be formed. The key cyclobutane intermediate 143 was itself formed via an elegant [2+2] cycloaddition strategy (Scheme 24).

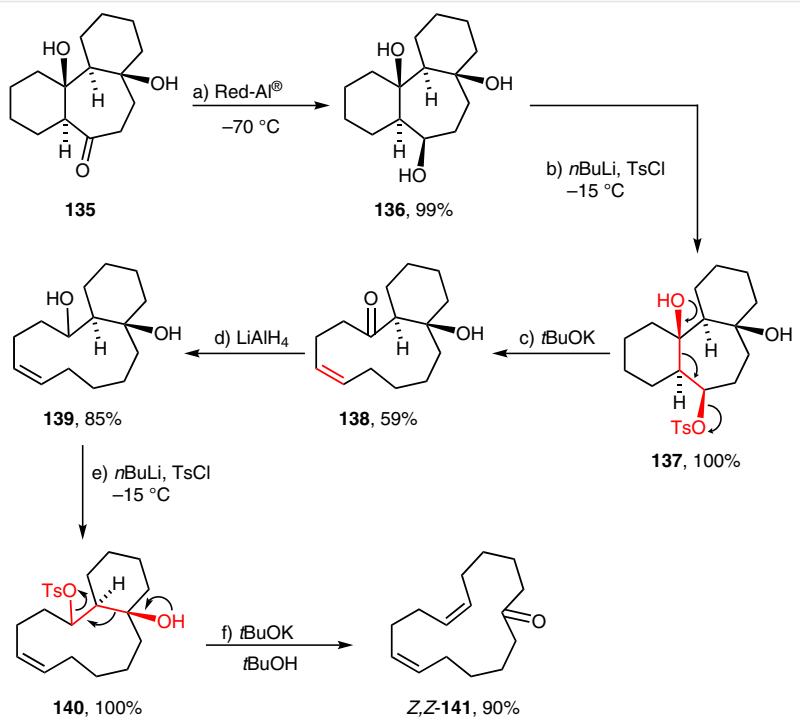

Scheme 23 A double Grob fragmentation sequence

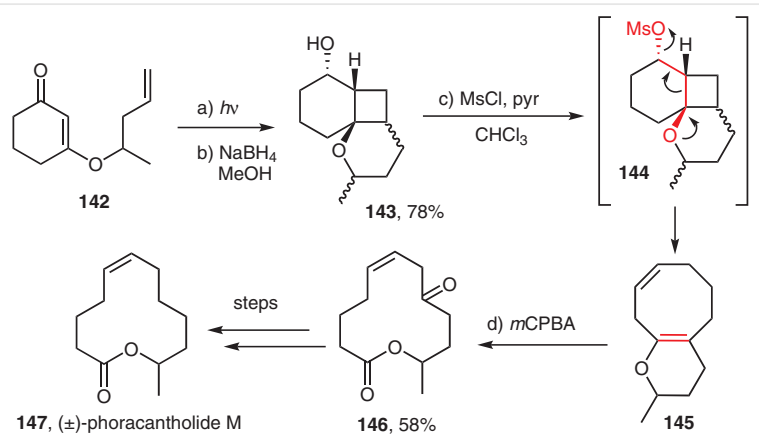

Scheme 24 A sequential Grob/oxidative fragmentation sequence

Maio and co-workers ${ }^{36}$ have reported that sequential ring expansions can be used to form medium-sized lactones, including the synthesis of natural product (-)-phoracantholide J in six linear steps and an overall $26 \%$ yield (Scheme 25). Thus, silyl enol ether 148 was treated with methyllithium to form the corresponding enolate and then reacted with epoxide in the presence of boron trifluoride to form hemiketal 149. Then, oxidative fragmentation was achieved using diacetoxyiodobenzene and iodine, to form geometrically pure cis-olefinic 8-membered lactone $\mathbf{1 5 0}$. Treatment with TBAF revealed primary alcohol $\mathbf{1 5 1}$ and this set up the second ring-expansion reaction, which occurred via a spontaneous translactonization to form hydroxy lactone 152; the thermodynamically favorable change in ring size (8- to 10-membered) likely facilitated this facile ring expansion. Radical deoxygenation was then used to complete the synthesis of (-)-phoracantholide J (not shown). The synthesis was performed on gram scale, demonstrating the scalability of this impressive reaction sequence. 


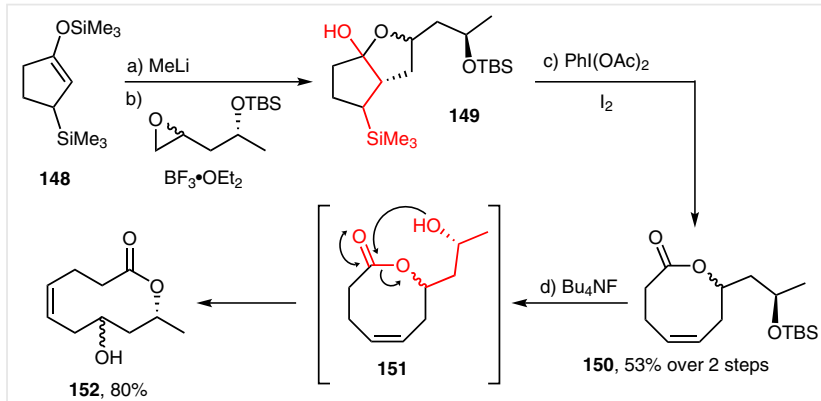

Scheme 25 Sequential ring expansions during the total synthesis of (-)-phoracantholide J

Murai and co-workers ${ }^{37}$ have reported an interesting successive ring-expansion reaction using bromo-diepoxides, promoted by AgOTf. As a note, this chemistry is different to the other examples described in this Account, in that the consecutive ring-expansion reactions do not take place on the same ring (meaning that it is not suitable for 'growing' medium-sized rings and macrocycles), but we decided to include it nonetheless as an example of a powerful ringexpansion cascade system. Thus, in a prototypical example of this method, epoxide $\mathbf{1 5 3}$ is activated by $\mathrm{Ag}(\mathrm{I})$ and undergoes 5-exo-tet cyclization. The resulting strained bicyclic epoxonium intermediate 154 then undergoes intramolecular cyclization from the second epoxide to form tetrahydropyran 155. An intermolecular nucleophilic attack then takes place, which leads to relief of ring strain and the formation of fused tetrahydropyran structure 156 (Scheme 26). Extensive studies have continued utilizing polyepoxide precursors and ring-expansion cascades to form multiple fused rings in this way, with this topic covered in a detailed review article. $^{38}$

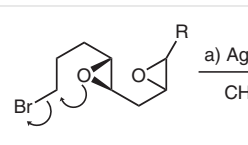

153

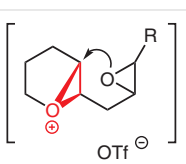

154

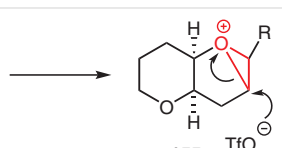

$155 \mathrm{~T}$<smiles>[R]C1OC2CCCOC2CC1(O)[Hg]</smiles>

Scheme 26 Successive ring-expansion cascade of bromo-diepoxide 153

Finally, Clayden and co-workers recently reported a method to access medium-sized rings via a three-atom ring expansion of metalated ureas via an insertion-type ring expansion, which was followed by a second ring expansion via an acid-catalyzed fragmentation reaction. ${ }^{39}$ Thus, treatment of indoline $\mathbf{1 5 7}$ with triphosgene furnished urea $\mathbf{1 5 8}$, which was then lithiated with LDA and DMPU (1,3-dimethyl-3,4,5,6-tetrahydro-2(1H)-pyrimidinone), promoting a migratory ring expansion to form ring-expanded urea 160. ${ }^{39 a}$ The reaction is driven by an increase in anion stability in migrating the negative charge from a benzylic carbanion position to a deprotonated urea group. DMPU is critical to the success of this process as it suppresses a competing 1,2 -acyl shift reaction. The transformation is stereospecific and proceeds with retention of configuration via a conformationally stable organolithium intermediate; a concerted associative mechanism is proposed, rather than a stepwise $\mathrm{S}_{\mathrm{N}} A$-type process. The same group later went on to show that cyclic ureas of the form $\mathbf{1 6 0}$ undergo facile ring-contraction reactions to form 1-aryl tetrahydroisoquinolines and tetrahydrobenzazepines (not shown) and in a single case, also showed that urea $\mathbf{1 6 0}$ could be ring-expanded for a second time following treatment with PTSA, to form 11membered 162 via the fragmentation mechanism shown in Scheme 27.39b

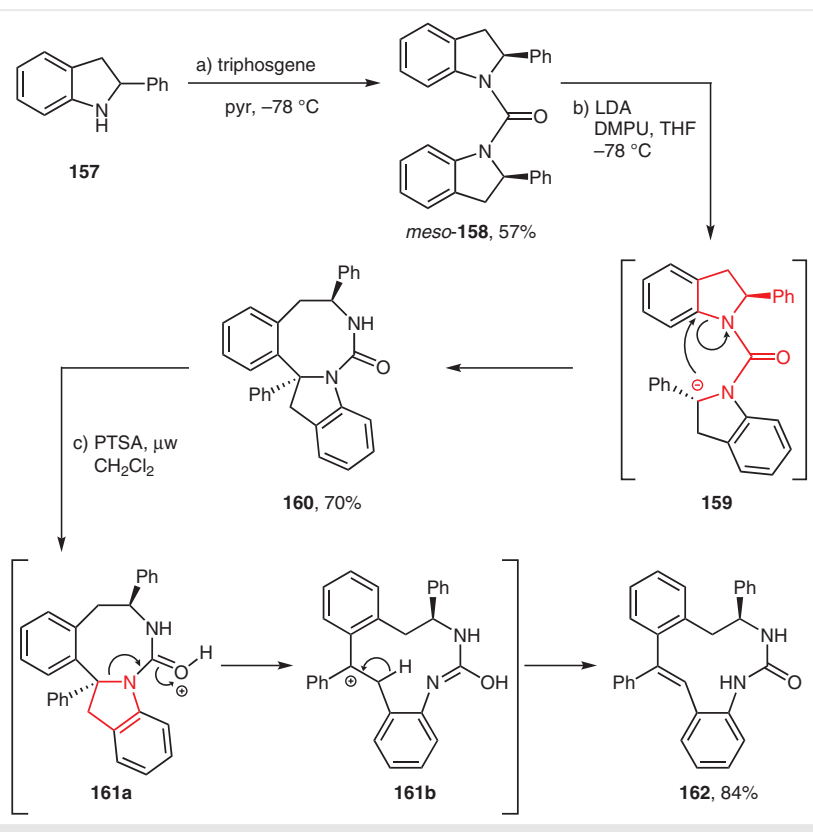

Scheme 27 Insertion/fragmentation double ring expansion of mesourea 158

\section{Conclusions and Future Outlook}

The importance of large ring molecules in a wide range of important applications means that the continued development of practical and scalable methods for their synthesis will always be of high value. ${ }^{1,2}$ Ring-expansion reactions have already proven themselves to be very useful in this regard, especially as they typically do not require the impractical reaction conditions, such as high dilution, that are often needed in typical end-to-end cyclization reactions. Although considerably less well developed than single ringexpansion processes, this review highlights the synthetic benefits of applying ring-expansion reactions consecutive- 
ly, with several of the examples being used either to generate natural products or scaffolds structurally related to bioactive macrocycles, for example, cyclic peptide mimics.

One of the major trends highlighted in this Account is that expanding a 'normal'-sized ring (5-7-membered) into a medium-sized ring (8-11-membered) is often more challenging than other ring-expansion processes in view of the relative instability of medium-sized rings. Conversely, the expansion of medium-sized to macrocycles is often much easier, as in this scenario moving away from the mediumsized scaffold to a more flexible, less strained macrocycle can bring thermodynamic advantages. In the context of consecutive ring expansions, especially starting from normal (5-7-membered) ring sizes, this can mean that while the first 'normal to medium' transformation can be challenging, once this barrier has been surpassed, other ringexpansion iterations will often become easier, which is useful to consider when designing consecutive sequences.

Arguably, the strategies that can be performed iteratively and with little or no extra transformations between the ring-expansion steps are the most useful and most likely to be widely adopted, especially if these methods allow for the versatile introduction of different functional groups in sequence. This Account also highlights several synthetic strategies in which two different classes of ring expansion have been performed in sequence to good effect. An interesting avenue that may be explored in the future is to design starting materials containing functionality compatible with two (or more) different ring-expansion methods, especially if each method can be performed orthogonally to the other in two directions.

\section{Funding Information}

The authors wish to thank the Leverhulme Trust (for an Early Career Fellowship, ECF-2015-013, for W. P. U.), the University of York (T. C. S. and W. P. U.), and the EPSRC (for a contribution to the DTA studentship for T. C. S., 1792616) for funding.

\section{References}

(1) For large rings in medicinal chemistry, see: (a) Driggers, E. M. Hale S. P.; Lee, J.; Terrett, N. K. Nat. Rev. Drug Discov. 2008, 7, 608. (b) Marsault, E. Peterson M. L. J. Med. Chem. 2011, 54, 1961. (c) Ghadiri, M. R.; Granja, J. R. Acc. Chem. Res. 2013, 46, 2955. (d) Giordanetto, F.; Kihlberg, J. J. Med. Chem. 2014, 57, 278. (e) Yudin, A. K. Chem. Sci. 2015, 6, 30.

(2) For large ring molecules in other applications, see: (a) Ema, T.; Tanida, D.; Sakai, T. J. Am. Chem. Soc. 2007, 129, 10591. (b) Griffiths, K. E.; Stoddart, J. F. Pure Appl. Chem. 2008, 80, 485. (c) Evans, N. H.; Beer, P. D. Chem. Soc. Rev. 2014, 43, 4658. (d) Xue, M.; Yang, Y.; Chi, X.; Yan, X.; Huang, F. Chem. Rev. 2015, 115, 7398. (e) Byrne, J. P. Blasco S.; Aletti, A. B.; Hessman, G.; Gunnlaugsson, T. Angew. Chem. Int. Ed. 2016, 55, 8938. (f) Sato, K.; Itoh, Y.; Aida, T. J. Am. Chem. Soc. 2011, 133, 13767. (g) Iyoda, M.; Yamakawa, J.; Rahman, M. J. Angew. Chem. Int. Ed. 2011, 50, 10522.
(3) For useful background and discussion on macrocyclization strategies in general, see: (a) Parenty, A.; Moreau, X.; Campagne, J. M. Chem. Rev. 2006, 106, 911. (b) Hill, R.; Rai, V.; Yudin, A. K. J. Am. Chem. Soc. 2010, 132, 2889. (c) White, C. J.; Yudin, A. K. Nat. Chem. 2011, 3, 509. (d) Saito, F.; Bode, J. W. Nat. Chem. 2016, 8, 1085. (e) Practical Medicinal Chemistry with Macrocycles; Marsault, E.; Peterson, M. L., Ed.; Wiley: Hoboken, NJ, 2017.

(4) (a) Illuminati, G.; Mandolini, L. Acc. Chem. Res. 1981, 14, 95. (b) Fastrez, J. J. Phys. Chem. 1989, 93, 2635. (c) Collins, J. C.; James, K. Med. Chem. Commun. 2012, 3, 1489.

(5) For pseudo-high-dilution methods (including the use of solid supported systems and phase separation), see: (a) Mazur, S.; Jayalekshmy, P. J. Am. Chem. Soc. 1979, 101, 677. (b) Treder, A. P.; Hickey, J. L.; Tremblay, M.-C. J.; Zaretsky, S.; Scully, C. C. G.; Mancuso, J.; Doucet, A.; Yudin, A. K.; Marsault, E. Chem. Eur. J. 2015, 21, 9249. (c) Rosenbaum, C.; Waldmann, H. Tetrahedron Lett. 2001, 42, 5677. (d) Bédard, A.-C.; Collins, S. K. J. Am. Chem. Soc. 2011, 133, 19976.

(6) (a) Haas, K.; Ponikwar, W.; Nöth, H.; Beck, W. Angew. Chem. Int. Ed. 1998, 37, 1086. (b) Gerbeleu, N. V.; Arion, V. B.; Burgess, J. P. In Template Synthesis of Macrocyclic Compounds; Wiley-VCH: Weinheim, 1999. (c) Fu, H.; Chang, H.; Shen, J.; Yu, L.; Qin, B.; Zhang, K.; Zeng, H. Chem. Commun. 2014, 50, 3582.

(7) Roesner, S.; Saunders, G. J.; Wilkening, I.; Jayawant, E.; Geden, J. V.; Kerby, P.; Dixon, A. M.; Notman, R.; Shipman, M. Chem. Sci. 2019, 10, 2465.

(8) Gartner, Z. J.; Tse, B. N.; Grubina, R.; Doyon, J. B.; Snyder, T. M.; Liu, D. R. Science 2004, 305, 1601.

(9) For an excellent account of classical ring-expansion approaches, see: (a) Ring Enlargement in Organic Chemistry; Hesse, M., Ed.; Wiley-VCH: Weinheim, 1991. For a more recent review, see: (b) Unsworth, W. P.; Donald, J. R. Chem. Eur. J. 2017, 23, 8780.

(10) For prominent recent examples of ring-expansion methods, see: (a) Li, Z.-L.; Li, X.-H.; Wang, N.; Yang, N.-Y.; Liu, X.-Y. Angew. Chem. Int. Ed. 2016, 55, 15100. (b) Li, L.; Li, Z.-L.; Wang, F.-L.; Guo, Z.; Cheng, Y. F.; Wang, N.; Dong, X.-W.; Fang, C.; Liu, J.; Hou, C.; Tan, B.; Liu, X.-Y. Nat. Commun. 2016, 7, 13852. (c) Mendoza-Sanchez, R.; Corless, V. B.; Nguyen, Q. N. N.; Bergeron-Brlek, M.; Frost, J.; Adachi, S.; Tantillo, D. J.; Yudin, A. K. Chem. Eur.J. 2017, 23, 13319. (d) Loya, D. R.; Jean, A.; Cormier, M.; Fressigné, C.; Nejrotti, S.; Blanchet, J.; Maddaluno, J.; De Paolis, M. Chem. Eur. J. 2018, 24, 2080. (e) Guney, T.; Wenderski, T. A.; Boudreau, M. W.; Tan, D. S. Chem. Eur. J. 2018, 24, 13150. (f) Osipyan, A.; Sapegin, A.; Novikov, A. S.; Krasavin, M. J. Org. Chem. 2018, 83, 9707. (g) Massaro, N. P.; Stevens, J. C.; Chatterji, A.; Sharma, I. Org. Lett. 2018, 20, 7585. (h) Kumar, P.; Dey, R.; Banerjee, P. Org. Lett. 2018, 20, 5163. (i) Reutskaya, E.; Osipyan, A.; Sapegin, A.; Novikov, A. S.; Krasavin, M. J. Org. Chem. 2019, 84, 1693. (j) Gao, X.; Xia, M.; Yuan, C.; Zhou, L.; Sun, W.; Li, C.; Wu, B.; Zhu, D.; Zhang, C.; Zheng, B.; Wang, D.; Guo, H. ACS Catal. 2019, 9, 1645. (k) Loya, D. R.; De Paolis, M. Chem. Eur. J. 2019, 25, 1842.

(11) Lehmann, J. W.; Blair, D. J.; Burke, M. D. Nat. Rev. Chem. 2018, For perspective on the importance of iterative synthetic methods, see: 2, 115; and references cited therein.

(12) For macrocyclic polymerisaton methods, see: (a) Kricheldorf, $H$. R.; Lee, S.-R.; Schittenhelm, N. Macromol. Chem. Phys. 1998, 199, 273. (b) Kudo, H.; Makino, S.; Kameyama, A.; Nishikubo, T. Macromolecules 2005, 38, 5964. (c) Kricheldorf, H. R. J. Polym. Sci. A Polym. Chem. 2010, 48, 251. (d) Kudo, H.; Takeshi, Y.J. Polym. Sci. A Polym. Chem. 2014, 52, 857. 
(13) For selected Hesse works, see ref. 9a and: (a) Kramer, U.; Guggisberg, A.; Hesse, M.; Schmid, H. Angew. Chem., Int. Ed. Engl. 1977, 16, 861. (b) Kramer, U.; Schmid, H.; Guggisberg, A.; Hesse, M. Helv. Chim. Acta 1979, 62, 811.

(14) Kramer, U.; Guggisberg, A.; Hesse, M.; Schmid, H. Angew. Chem., Int. Ed. Engl. 1978, 17, 200.

(15) Veith, H. J.; Hesse, M.; Schmid, H. Helv. Chim. Acta 1970, 53, 1355.

(16) Kimura, E.; Koike, T.; Takahashi, M.J. Chem. Soc., Chem. Commun. 1985, 385.

(17) Corey, E. J.; Brunelle, D. J.; Nicolaou, K. C. J. Am. Chem. Soc. 1977, 99, 7359.

(18) (a) Tam, J. P.; Lu, Y.-A.; Yu, Q. J. Am. Chem. Soc. 1999, 121, 4316. (b) Hemu, X.; Qiu, Y.; Tam, J. P. Tetrahedron 2014, 70, 7707.

(19) Pattenden, G.; Schulz, D. J. Tetrahedron Lett. 1993, 34, 6787.

(20) Fouque, E.; Rousseau, G.; Seyden-Penne, J. J. Org. Chem. 1990, $55,4807$.

(21) Kitsiou, C.; Hindes, J. J.; I'Anson, P.; Jackson, P.; Wilson, T. C.; Daly, E. K.; Felstead, H. R.; Hearnshaw, P.; Unsworth, W. P. Angew. Chem. Int. Ed. 2015, 54, 15794.

(22) Baud, L. G.; Manning, M. A.; Arkless, H. L.; Stephens, T. C.; Unsworth, W. P. Chem. Eur. J. 2017, 23, 2225.

(23) Stephens, T. C.; Lodi, M.; Steer, A. M.; Lin, Y.; Gill, M. T.; Unsworth, W. P. Chem. Eur. J. 2017, 23, 13314.

(24) Stephens, T. C.; Lawer, A.; French, T.; Unsworth, W. P. Chem. Eur. J. 2018, 24, 13947.

(25) Vedejs, E.; Reid, J. G. J. Am. Chem. Soc. 1984, 106, 4617.

(26) Vedejs, E.; Hagen, J. P. J. Am. Chem. Soc. 1975, 97, 6878.
(27) Schmid, R.; Schmid, H. Helv. Chim. Acta 1977, 60, 1361.

(28) (a) Vedejs, E.; Mullins, M. J.; Renga, J. M.; Singer, S. P. Tetrahedron Lett. 1978, 19, 519. (b) Vedejs, E. Acc. Chem. Res. 1984, 17, 358.

(29) Weston, M. H.; Nakajima, K.; Back, T. G. J. Org. Chem. 2008, 73, 4630.

(30) (a) Lee, Y.-S.; Jung, J.-W.; Kim, S.-H.; Jung, J.-K.; Paek, S.-M.; Kim, N.-J.; Chang, D.-J.; Lee, J.; Suh, Y.-G. Org. Lett. 2010, 12, 2040. (b) Suh, Y.-G.; Kim, S.-A.; Jung, J.-K.; Shin, D.-Y.; Min, K.-H.; Koo, B.-A.; Kim, H.-S. Angew. Chem. 1999, 111, 3753.

(31) Suh, Y.-G.; Lee, Y.-S.; Kim, S.-H.; Jung, J.-K.; Yun, H.; Jang, J.; Kim, N.-J.; Jung, J.-W. Org. Biomol. Chem. 2012, 10, 561.

(32) Jung, J.-W.; Kim, S.-H.; Suh, Y.-G. Asian J. Org. Chem. 2017, 6, 1117.

(33) Zhang, W.; Dowd, P. Tetrahedron Lett. 1996, 37, 957.

(34) Fehr, C.; Galindo, J.; Etter, O.; Thommen, W. Angew. Chem. Int. Ed. 2002, 41, 4523.

(35) Ikdea, M.; Ohno, K.; Takahashi, M.; Homma, K.-I.; Uchino, T.; Tamura, Y. Heterocycles 1983, 20, 1005.

(36) Posner, G. H.; Hatcher, M. A.; Maio, W. A. Org. Lett. 2005, 7, 4301.

(37) Hayashi, N.; Fujiwara, K.; Murai, A. Tetrahedron Lett. 1996, 37, 6173.

(38) Fujiwara, K.; Murai, A. Bull. Chem. Soc. Jpn. 2004, 77, 2129.

(39) (a) Hall, J. E.; Matlock, J. V.; Ward, J. W.; Gray, K. V.; Clayden, J. Angew. Chem. Int. Ed. 2016, 55, 11153. (b) Hill, J. E.; Matlock, J. V.; Lefebvre, Q.; Cooper, K. G.; Clayden, J. Angew. Chem. Int. Ed. 2018, 57,5788 